\title{
Caracterização e análise do estado mental e funcional de idosos institucionalizados em instituições públicas de longa permanência
}

\author{
Analysis and characterization of functional capacity \\ and mental state in residents in old folk's home \\ Maria Estelita Rojas Converso', Isabele lartelli'
}

\section{RESUMO}

Objetivo: $O$ estudo objetivou caracterizar os idosos institucionalizados residentes em três instituições de longa permanência para idosos, quanto a sua capacidade funcional e seu estado mental, e verificar se existe correlação entre ambas. Métodos: Para isso foram utilizados três instrumentos: um formulário de identificação, o Mini-Exame do Estado Mental (MEEM) e o Índice de Barthel. Foram utilizados como tratamento estatístico a Correlação de Person e o Teste da ANOVA. Resultados: A população avaliada consistiu de 115 idosos com idades entre 62 e 104 anos, sendo $40,66 \%$ do sexo feminino e 59,13\% do sexo masculino. São alfabetizados $49,56 \%$ e analfabetos $50,43 \%$, quanto ao estado civil predominaram os solteiros $(46,65 \%)$ e viúvos (21,8\%). Quanto ao estado mental e funcional, houve alta taxa de idosos apresentando déficit cognitivo (76,72\%) e de idosos independentes funcionalmente (75,65\%). Conclusões: De acordo com os resultados observa-se que existe correlação significativa entre o Índice de Barthel e o MEEM ( $r=0,441 ; p<0,000)$, e também que as variáveis sexo e idade não influen-

\section{Palavras-chave}

Idoso, demência, MEEM.

\section{Key-words}

Elderly, dementia, MMSE.

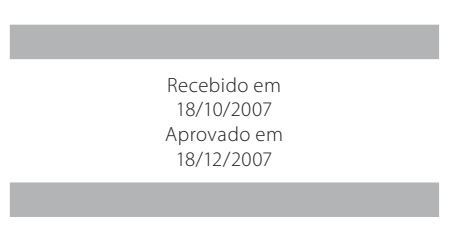

ciam nos resultados destes instrumentos. Existe relação estatística significativa $(p<0,0001)$ entre escolaridade e estado mental e/ou funcional do indivíduo.

\section{ABSTRACT}

Background: This study had as objective to characterize the institutionalized elderly residents in three long-term care facilities, about their functional capacity and mental state, and to evaluate if there is a correlation between both. Methods: To do so, three instruments were used: an identification form, the Mini-Mental State Examination (MMSE) and Barthel's Index. For statistical analysis the Person's Correlation and ANOVA Test were used. Results: The population studied consisted of 115 elders with ages between 62 and 104 years old, 40.66\% females and $59.13 \%$ males. $49.56 \%$ are literate and $50.43 \%$ illiterate. Regarding the marital status, singles (46.65\%) and widows (21.8\%) were predominant. About the mental and functional state, there was a high rate of elders showing cognitive decline (76.72\%) and functionally independent (75.65\%). Conclusions: According to the results, one can notice that there is a mild degree of correlation between the Barthel's Index and MMSE ( $r=0.441 ; p<0.000)$, and the variables of gender and age are not influenced in the results of these instruments. There is a significant statistical relation $(p<0.0001)$ between education and mental and/or functional state of the individual.

1 Departamento de Fisioterapia da Universidade Estadual Paulista "Júlio de Mesquita Filho" (Unesp) e da Faculdade de Ciências e Tecnologia (FCT/Unesp) - Campus de Presidente Prudente.

Endereço para correspondência: Maria Estelita Rojas Converso

Rua Dirce Macuco Sandoval, 255 - 19053-670 - Presidente Prudente, SP

Fone.: (18) 3229-5365. E-mail: converso@fct.unesp.br 


\section{INTRODUÇÃO}

Atualmente as pesquisas sobre envelhecimento vêm ganhando cada vez mais espaço no mundo científico, por causa do progressivo aumento do número de idosos no mundo (Prado e Sayd, 2004). De acordo com o censo 2000 (IBGE), o Brasil possui 14.536.029 indivíduos acima de 60 anos, o que corresponde a 8,6\% da população total brasileira. Em Presidente Prudente/SP, o número de idosos é de 19.491, ou seja, 10\% da população total da cidade. Grande parte desses idosos apresenta problemas associados ao envelhecimento, tendo implicações importantes na sua própria vida, de sua família e da comunidade em que vive.

Apesar disso os órgãos responsáveis pela assistência social e de saúde no Brasil ainda não possuem uma política consistente para lidar com esses problemas (Carvalho Filho e Papaléu Netto, 2000; Garrido e Menezes, 2002).

Muitos foram os fatores que impediram o processo de conhecimento do envelhecimento, principalmente o fato de caracterizá-lo erroneamente como um processo patológico, estimulando mais a tentativa de combatê-lo do que entendê-lo (Carvalho Filho e Papaléu Netto, 2000).

De acordo com alguns autores, o envelhecimento pode ser conceituado como um processo dinâmico e progressivo ocorrendo modificações morfológicas, funcionais, bioquímicas e psicológicas, com ritmo e intensidade diferentes para cada indivíduo, que determinam progressiva perda da capacidade de adaptação do indivíduo ao meio ambiente, ocasionando maior vulnerabilidade e maior incidência de processos patológicos que terminam por levá-lo à morte (Carvalho Filho e Papaléu Netto, 2000; Jordão Netto, 1997; Vieira Brandão, 1995).

Quando as funções começam a deteriorar, como resultado de uma demanda excessiva imposta a um sistema fisiologicamente incapaz de supri-la e/ou pela existência de processos patológicos (camuflados ou não), é que os problemas começam a surgir. O conceito está intimamente ligado à manutenção da autonomia. Nesse sentido, portanto, o conceito de envelhecimento cronológico passa a ser de relevância muito menor que o conceito do envelhecimento funcional (Kalache et al., 2000; Cançado, 1994; CarvaIho Filho e Papaléu Netto, 2000).

O sistema nervoso central é o sistema mais comprometido com o processo do envelhecimento, sendo atingido tanto por fatores intrínsecos (genéticos) como extrínsecos (meio ambiente, metabolismo, radicais livres etc.), afetando, conseqüentemente, as funções mais nobres do organismo, como aquelas que capacitam o indivíduo para a vida social, diminuindo a capacidade intelectual, no sentido mais amplo possível, com alterações da memória, raciocínio lógico, juízo crítico, funções práxicas e gnósicas, na orientação do espaço, na fala e outras formas de comunicação, e ainda na afetividade, na personalidade e na conduta. Entretanto, todos os sinais de deficiências neurológicas presentes na senilidade (envelhecimento patológico) podem não ocorrer na senescência (envelhecimento sadio) ou, se presentes, serão com menor intensidade, tanto na diversidade quanto na perda da capacitação de vida social. Na maioria dos idosos, essas deficiências ocorrem tão lentamente, que a própria estimulação psicossocial-afetiva é capaz de superar esses possíveis déficits neurofuncionais (Cançado, 1994).

Uma importante afecção que atinge os idosos, e que vem sendo muito estudada atualmente, é a demência, caracterizada pela diminuição global das funções cognitivas, embora não necessariamente de modo uniforme, associada a um estado preservado da consciência (Carvalho Filho e Papaléu Netto, 2000). Ela pode resultar de uma variedade de condições degenerativas, vasculares, neoplásicas, infecciosas, tóxicas, metabólicas e psiquiátricas, que estarão localizadas em diferentes partes do cérebro, afetando diferentes funções cognitivas (Vieira Brandão, 1995). Atinge cerca de 10\% a 15\% dos indivíduos acima de 65 anos nos seus mais variados graus. Deste grupo, a demência mais freqüente é a doença de Alzheimer, atingindo 50\% a 60\% destes indivíduos (Cançado, 1994).

A doença de Alzheimer caracteriza-se inicialmente por um ligeiro declínio das capacidades intelectuais, como o prejuízo da memória recente, dificuldade de manter a atenção, a capacidade de associação e a orientação, sobretudo espacial, seguido de lentidão do pensamento e da capacidade de executar tarefas sociais e econômicas. Começa a haver um declínio geral da eficiência, apresentando uma aparência descuidada, distúrbios de movimentos e de postura, em razão da fraqueza e rigidez dos músculos. Os distúrbios da fala são precoces: anomia, dificuldade para entender o que está escrito ou o que é falado, ecolalia (repetição de frases) e verbigeração. Há ainda várias apraxias e vários tipos de agnosias. Na fase terminal, o paciente passa a uma existência vegetativa e a apresentar um semblante inexpressivo e total deterioração intelectual e motora (Cançado, 1994; Vieira Brandão, 1995; Rosenfeld, 2002).

Nesse processo evolutivo de degeneração, torna-se impossível viver sem apoio de terceiros, uma tarefa difícil em razão da total dependência física do paciente e da inviabilidade de diálogo e qualquer forma de expressão deste. Este cuidado integral causa desgaste da família que vivencia uma verdadeira revolução domiciliar, levando na maioria das vezes a institucionalização do idoso (Vieira Brandão, 1995; Sociedade Brasileira de Geriatria e Gerontologia, 1994; Garrido e Almeida, 1999).

Alguns autores definem a instituição como instrumento de controle e de utilização da energia social sendo, ao mesmo tempo, uma estrutura decorrente de necessidades sociais, e que possui alto custo e é de difícil manutenção. É por tudo isso que, atualmente, a internação de idosos está 
sendo posta em questão até pelos países desenvolvidos, onde estes serviços são sofisticados e oferecem grande conforto e eficiência. Entretanto, quando a família não consegue manter um nível adequado de cuidado ou quando não dispõe de nenhum membro que tenha tempo ou disponibilidade interna para desempenhar a tarefa, a primeira alternativa tem sido a contratação de cuidadores profissionais, para desenvolver os cuidados que o idoso necessita, e a outra seria encaminhá-lo para uma instituição, como asilos ou casas geriátricas (Vieira Brandão, 1995; Karsch, 2003; Salgado, 1982).

Por causa de todos esses aspectos é que se objetivou caracterizar os idosos institucionalizados em instituições de longa permanência (ILP) públicas da cidade de Presidente Prudente quanto a sua capacidade funcional e seu estado mental e verificar a relação entre a capacidade funcional e o estado mental desses idosos.

\section{MÉTODO}

Inicialmente foi enviado um ofício às ILPs solicitando permissão para a realização do trabalho, somente depois iniciou-se a coleta de dados. O projeto de pesquisa foi aprovado pelo Comitê de Ética em Pesquisa da Faculdade de Ciências e Tecnologia da Universidade Estadual Paulista "Júlio de Mesquita Filho" (FCT/Unesp), conforme Processo no 12/2005.

A amostra foi composta por 115 idosos de três ILP de Presidente Prudente: Hospital Psiquiátrico Espírita Adolpho Bezerra de Menezes (Hopebeme), Vila da Fraternidade e Lar São Rafael.

A coleta de dados consistiu da aplicação de três instrumentos:

- Formulário: composto por questões de identificação do idoso. O preenchimento deste instrumento se deu por meio da consulta aos prontuários dos idosos. Apenas uma das ILP não permitiu a consulta aos prontuários, e as informações foram colhidas diretamente com os idosos.

- Índice de Barthel (Mahoney e Barthel, 1965): questionário utilizado para verificar a situação funcional dos indivíduos. O protocolo é composto por dez áreas de atividades de vida diária (AVDs) que incluem alimentação, transferência, toalete pessoal, uso do banheiro, andar em superfície plana, propulsão de cadeira de rodas, subir e descer escadas, vestir e despir-se e controles dos esfíncteres anal e vesical. O resultado total varia entre 0 a 100 pontos, variando de dependentes e independentes. As informações para este instrumento deram-se pela observação da própria pesquisadora e pelo questionamento dos cuidadores dos idosos.

- Mini-Exame do Estado Mental (MEEM): instrumento de rastreio de comprometimento cognitivo, podendo ser utilizado na detecção de perdas cognitivas. O MEEM foi desenvolvido por Folstein e McHugh em 1975 e traduzido por Bertolucci et al. (1994), é composto por diversas questões tipicamente agrupadas em sete categorias, cada uma delas desenhada com o objetivo de avaliar funções cognitivas específicas: orientação para tempo (5 pontos), orientação para local (5 pontos), registro de três palavras (3 pontos), atenção e cálculo (5 pontos), lembrança das três palavras (3 pontos), linguagem (8 pontos) e capacidade construtiva visual (1 ponto). O escore do MEEM pode variar de um mínimo de 0 até um total máximo de 30 pontos. Entretanto, de acordo com Almeida (1998) os escores do MEEM sofrem influência significativa da idade e da escolaridade do indivíduo, sugerindo a necessidade de se utilizar pontos de corte diferenciados. Para idosos sem escolaridade o ponto de corte mais adequado para o diagnóstico de demência deve ser 19, e para os idosos com alguma instrução escolar o ponto de corte deve ser 23 (Laks et al., 2003; Almeida, 1998). Este instrumento foi aplicado pela pesquisadora, diretamente aos idosos, seguindo as normas propostas por Folstein.

Para a análise dos dados foram utilizados o método estatístico descritivo simples, a Correlação de Person e o Teste da ANOVA. O nível de significância para todos os testes foi fixado em 0,05.

\section{RESULTADOS}

Dos 115 indivíduos analisados 40,66\% eram mulheres e 59,13\% homens, com idade compreendida entre 62 e 104 anos. Desses, 50,43\% eram analfabetos e 49,56\% alfabetizados.

Quanto ao estado civil 17,6\% eram casados, $46,55 \%$ solteiros, 21,73\% viúvos e 13,52\% divorciados.

Em relação ao estado mental avaliado pelo MEEM encontramos $76,72 \%$ com déficit cognitivo e 23,28\% normais. Além disso, o escore médio para este instrumento foi de 13 pontos (Tabela 1), confirmando os resultados obtidos.

O Índice de Barthel avaliou o estado funcional dos idosos, visto que quanto maior fosse a pontuação obtida pelo indivíduo, mais independente em suas AVDs ele seria. O resultado total do Índice de Barthel da população estudada foi de 11,32\% de 0 a 25 pontos; 4,34\% de 26 a 50 pontos; 8,69\% de 51 a 75 pontos e 75,65\% de 76 a 100 pontos. Os resultados mostraram grande número de idosos com pontuação acima de 76 pontos (75,65\%), implicando um bom estado funcional e/ou independência para suas AVDs. A média para o escore deste instrumento foi de 80 pontos, confirmando a alta pontuação desta população.

Comparando-se os resultados do MEEM e do Índice de Barthel entre os sexos, pudemos observar que não há uma 
relação estatisticamente significativa (Tabela 1). Assim, podemos dizer que o estado mental e funcional não é diferenciado entre homens e mulheres.

Tabela 1. Representação das médias do MEEM e do Índice de Barthel em relação ao sexo da população

\begin{tabular}{lccc}
\hline \multirow{2}{*}{ Variável } & \multicolumn{2}{c}{ Sexo } & \multirow{2}{*}{ P-valor } \\
\cline { 2 - 3 } & Feminino & Masculino & \\
\hline MEEM & 11,681 & 13,926 & 0,2222 \\
Barthel & 80,745 & 79,926 & 0,8919 \\
\hline
\end{tabular}

Avaliando-se a influência da escolaridade nos instrumentos, vimos que existe uma relação estatisticamente significativa (Tabela 2), ou seja, a escolaridade pode influenciar no estado mental e/ou funcional do indivíduo.

Tabela 2. Representação das médias do MEEM, Índice de Barthel e idade em relação à escolaridade da população

\begin{tabular}{lccc}
\hline \multirow{2}{*}{ Variável } & \multicolumn{2}{c}{ Escolaridade } & \multirow{2}{*}{ P-valor } \\
\cline { 2 - 3 } & Alfabetizado & Analfabeto & \\
\hline MEEM & 18,842 & 7,276 & $<0,0001$ \\
Barthel & 92,632 & 68,103 & $<0,0001$ \\
\hline
\end{tabular}

Quanto ao estado civil encontramos que não existe uma relação com os instrumentos, como pode ser visto na Tabela 3, o que mostra que o estado mental e o estado funcional não sofrem influência em indivíduos com estado civil diferentes.

Tabela 3. Representação das médias do MEEM, Índice de Barthel e idade em relação ao estado civil da população

\begin{tabular}{lccccc}
\hline \multirow{2}{*}{ Variável } & \multicolumn{4}{c}{ Estado civil } & \multirow{2}{*}{ P-valor } \\
\cline { 2 - 5 } & Solteiro & Casado & Divorciado & Viúvo & \\
\hline MEEM & 9,796 & 12,050 & 18,813 & 17,000 & 0,0007 \\
Barthel & 71,111 & 77,250 & 89,063 & 96,800 & 0,0039 \\
\hline
\end{tabular}

De acordo com a Correlação de Person, encontramos que a idade não influencia nos instrumentos, no entanto existe uma correlação de grau moderado entre o MMSE e o Índice de Barthel ( $p<0,000)$, ou seja, o estado funcional sofre interferência do mental e vice-versa.

Tabela 4. Representação da Correlação de Person entre o MEEM, o Índice de Barthel e a idade da população

\begin{tabular}{lcc}
\hline & MEEM & Barthel \\
\hline \multirow{2}{*}{ Barthel } & 0,441 & \\
& 0,000 & \\
\multirow{2}{*}{ Idade } & 0,025 & 0,032 \\
& 0,791 & 0,737 \\
\hline
\end{tabular}

\section{DISCUSSÃO}

Segundo Leal et al. (1998), o número de idosos abandonados tem crescido muito rapidamente desde o início da década de 90 do século passado, representando mais de 300 miIhões da população mundial. Apesar de os dispositivos legais enfatizarem que o cuidado dos pais durante a doença, carência ou velhice cabe aos filhos, não há como se exigir destes o cumprimento desse encargo quando nem mesmo a sociedade assume sua parcela de responsabilidade, provendo recursos e criando condições para que o idoso possa permanecer em ambiente familiar. Muitos dos idosos residentes nas instituições analisadas não possuem qualquer responsável conhecido, e boa parcela deles foi abandonada pela família com a alegação de que não teria recursos financeiros para promovê-los da assistência necessária. Esta seria uma possível explicação para a maior incidência de idosos solteiros e viúvos encontrados no presente estudo, e que também são compatíveis aos resultados de Vieira (2004) ao analisar instituições filantrópicas e particulares de Florianópolis/SC.

Chaimowics e Greco (1999) caracterizaram idosos institucionalizados em Belo Horizonte e mostraram em seu estudo que 58,2\% dos idosos são solteiros, 22,4\% casados, $7,5 \%$ divorciados e 11,9\% são viúvos, dados que coincidem parcialmente com nossos resultados, em que o predomínio foi de idosos solteiros e viúvos.

Em relação à idade, Chaimowics e Greco (1999) encontraram que $81,1 \%$ das mulheres constituíam os asilos do município de Belo Horizonte, definiram que além do fato de as mulheres viverem mais do que os homens, ficam viúvas mais cedo, apresentando dificuldade para casar ou para casar novamente após a separação ou a viuvez. Dado esse não confirmado no presente estudo, no qual apenas $40,66 \%$ eram mulheres.

Quanto ao grau de escolaridade, Vieira (2004) ao analisar instituições filantrópicas e particulares de Florianópolis, encontrou uma prevalência do grupo de analfabetos, cerca de $62 \%$ da população, enquanto em nossa população tivemos $50,45 \%$ de analfabetos.

Segundo Schuling et al. (1993), a atividade funcional compreende a habilidade de a pessoa desempenhar tarefas no dia-a-dia, incluindo aspectos físicos, psicológicos e sociais. Essas atividades revelam a capacidade de a pessoa cuidar de si mesma, executar seus papéis e suas tarefas básicas e sociais.

Burgio (1991) afirma que a perda do funcionamento adaptativo em muitos idosos institucionalizados não é unicamente o resultado de um declínio ou de mudanças biológicas negativas, mas, sim, conseqüência de um ambiente que estabelece e decide a ocasião para o comportamento deficitário e que reforça o comportamento ineficaz e de dependência. Ramos et. al. (1993) encontraram que 47\% dos 
idosos moradores da área metropolitana na região sudeste do Brasil possuem perda da autonomia, 27\% são casos psiquiátricos e 86\% possui alguma doença crônica.

Ao utilizar o Índice de Katz em sua população, Vieira (2004) encontrou que cerca de 30\% foi considerada independente. Resultado diferente foi encontrado em nosso estudo utilizando o Índice de Barthel, no qual 75,65\% dos idosos residentes nas três instituições analisadas apresentaram pontuação acima de 76 pontos no Índice de Barthel, sendo considerados independentes funcionalmente. Consoante Valverde et al. (1994), as pontuações com 60 ou mais pontos são compatíveis com a vida em comunidade, desde que sejam excluídas as limitações impostas às atividades instrumentais da vida diária.

Apesar de os idosos analisados no presente estudo terem obtido pontuação alta no Índice de Barthel, encontrouse alta porcentagem de idosos com déficit cognitivo, o que pode explicar a sua institucionalização.

Segundo Ratey (2002), o movimento é a base da aprendizagem, porque representa um importante aspecto da experiência em cada momento, e aprender uma competência motora requer o desenvolvimento e a modificação de programas motores cada vez mais exatos, os quais coordenem não apenas movimentos precisos, mas também o conhecimento necessário para executá-los. Assim, podemos pensar o quanto os estados mental e funcional são dependentes, podendo um interferir no outro, como ocorre com os idosos analisados nesta pesquisa.

Engelhardt et al. (1997) acreditam que o diagnóstico de comprometimento cognitivo é tarefa complexa e ainda não bem sistematizada na população de idosos. Os quadros leves de comprometimento cognitivo são freqüentes, passando muitas vezes despercebidos, e há necessidade de distinguir, o que muitas vezes é difícil, entre manifestações iniciais de algumas doenças e modificações associadas com o processo normal do envelhecimento. De acordo com Capurso (1999), o declínio das funções cognitivas relacionadas com a idade refere-se geralmente deteriorização no desempenho da memória, nas funções executivas e na velocidade do processo cognitivo, sendo este um processo normal do envelhecimento. No entanto, quando o declínio dessas funções interfere na independência do idoso e em sua vida social, podemos estar lidando com uma situação patológica. No presente estudo, encontramos 76,72\% dos idosos apresentando déficit cognitivo, isso mostra o quão forte apresenta-se esta perda cognitiva nesta população.

A hipótese de que mais estímulo intelectual propicia um cérebro mais flexível na velhice é apoiada por Evans (1996), que estudou moradores idosos na comunidade operária americana, que foram submetidos a uma série de testes para avaliação das condições motoras, mentais e de memória, sendo repetidas as provas três anos depois. Os moradores que tinham menor grau de instrução mostraram maior declínio nos escores dos testes, independentemente de idade, local de nascimento, profissão, renda ou língua materna. Os idosos avaliados no presente estudo eram 50,43\% analfabetos e os demais tinham poucos anos de escolaridade, o que pode explicar a alta porcentagem de déficit cognitivo nesta população.

O envelhecimento não é somente um momento na vida do indivíduo, mas um processo extremamente complexo, que tem implicações tanto para a pessoa que o vive como para a sociedade que o assiste (Fraiman, 1991). Por isso, a importância de se estudar mais a fundo o perfil da população idosa brasileira para se definir estratégias de melhora de saúde e qualidade de vida.

\section{CONCLUSÃO}

O presente estudo mostrou que a população de idosos residentes nas três entidades avaliadas caracteriza-se, em sua maioria, por indivíduos solteiros (46,55\%), com idade média de 73 anos, sem alfabetização (50,43\%) e com predominância do sexo masculino (59,13\%). Quanto ao estado mental e funcional, houve alta taxa de idosos apresentando possível demência $(76,72 \%)$ e de idosos independentes funcionalmente (75,65\%), o que demonstra que não é só a falta de autonomia que determina a institucionalização do idoso, mas os aspectos cognitivos são, também, levados em consideração pelos familiares e/ou responsáveis.

As variáveis sexo, idade e estado civil não tiveram influência sobre o Índice de Barthel e o MEEM, no entanto, encontramos uma relação de dependência destes instrumentos com a escolaridade dos idosos.

Observou-se também que o Índice de Barthel e o MEEM apresentam correlação de grau moderado entre si, mostrando que os estados mental e funcional possuem dependência estaticamente significativa.

\section{REFERÊNCIAS}

Almeida OP. Mini-exame do estado mental e o diagnóstico de demência no Brasil. Arq. NeuroPsiquiatr. 56(3-B):605-12, 1998.

Bertolucci PHF, Brucki SMD, Campacci SR, Juliano Y. 0 mini-exame do estado mental em uma população geral: impacto da escolaridade. Arq. Neuropsiquiatric. 52(1):1-7, 1994.

Burgio LD. Behavioral staff training and management in geriatric long-term care facilities. In: Wisocki PA, editor. Handbook of clinical behavior therapy with the elderly client. New York: Plenum, 1991.

Cançado FAX. Noções práticas de geriatria. Belo Horizonte: Coopermed; 1994.

Capurso A. Estudo longitudinal italiano sobre o envelhecimento. 1999. [acesso em 2005 Sep 26]. Disponível em http://europa.eu.int/comm/agriculture/prom/olive/medinf/pt/media/ press8.htm

Carvalho Filho ET, Papaleo Netto M. Geriatria: fundamentos, clínica e terapêutica. São Paulo: Atheneu, 2000 
Chaimowicz F, Greco DB. Dinâmica da institucionalização de idosos em Belo Horizonte. Rev Saúde Pública. 33(5):454-60, 1999.

Engelhardt E, Laks J, Rozanthal M, von Poser NAS, Menkes C, Marinho VM. Idosos velhos ("oldest old"): rastreamento cognitivo com o MMSE. Rev Bras Neurol. 33(4):201-6, 1997.

Evans D. Search for the tourette syndrome and human behavior genes. Califórnia: Hope Press, 1996. Fernandes FS. 0 que pode a cidade fazer pelos idosos. Maturidade. 6:11-18, 1990

Fraiman AP. Coisa da idade. São Paulo: Hermes, 1991.

Garrido R, Almeida OP. Distúrbios de comportamento em pacientes com demência: impacto sobre a vida do cuidador. Arq Neuro-Psiquiatr. 57(2-B):427-34, 1999.

Garrido R, Menezes PR. 0 Brasil está envelhecendo: boas e más notícias por uma perspectiva epidemiológica. Rev Bras Psiquiatr. 24(Supl I):3-6, 2002.

IBGE, Fundação do Instituto Brasileiro de Geografia e Estatística, censo demográfico 2000. Rio de Janeiro: 2004

Jordão Netto A. Gerontologia básica. São Paulo: Lemos Editorial, 1997.

Kalache A, Veras RP, Ramos LR. 0 envelhecimento da população mundial: um desafio novo. Rev Saúde Pública. 21(3): 200-10, 1987.

Karsch UM. Idosos dependentes: famílias e cuidadores. Cad. Saúde Pública. Rio de Janeiro, 19(3):861-6, 2003.

Laks J, Batista EMR, Guilherme ERL, Contino ALB, Faria MEV, Figueira I, et al. 0 mini-exame do estado mental em idosos de uma comunidade: dados parciais de Santo Antônio de Pádua. Arq Neuro-Psiquiatr. 61(3-B):782-5, 2003.

Leal AA, Monteiro RAS, Bandeira KMB. A terceira idade: da rejeição familiar à procura de um novo lar. Anais do I Congresso Norte-Nordeste de Psicologia. Salvador, Bahia; 1998.
Mahoney Fl, Barthel DW. Functional evaluation: the Barthel Index. Md State Med J. 15:61-5, 1965.

Prado SD, Sayd JD. A pesquisa sobre envelhecimento humano no Brasil: grupos e linhas de pesquisa. Ciência \& Saúde Coletiva. 9(1):57-68, 2004.

Ramos LR, Rosa TEC, Oliveira ZM, Medina MCG, Santos FRG. Perfil do idoso em área metropolitana na região sudeste do Brasil: resultados de inquérito domiciliar. Rev Saúde Pública. 27(2):87-94, 1993.

Ratey JJ. 0 cérebro - um guia para usuário: como aumentar a saúde, agilidade e longevidade de nossos cérebros através das mais recentes descobertas científicas. Rio de Janeiro: Objetiva, 2002.

Rosenfeld I. Viva agora envelheça depois: maneiras comprovadas de desacelerar o tempo. São Paulo: Ed. UNESP, 2002.

Salgado MA. A velhice uma questão social. São Paulo: Sesc: Centros de Estudos da terceira Idade, 1982.

Schuling J, de Haan R, Limburg M, Groenier H. The frenchay activites index: assessment of functional status in stroke patients. Stroke, 24:1173-7, 1993.

Sociedade Brasileira de Geriatria e Gerontologia. Caminhos do envelhecer. Rio de Janeiro: Revinter, 1994.

Valverde MD, Flórez M, Sánchez I. Escala de actividades de la vida diária. Rehabilitación. 28 : 377-88, 1994.

Vieira Brandão E. Manual de gerontologia. São Paulo: Papirus, 1995.

Vieira GF. Estudos dos parâmetros motores de idosos residentes em instituiç̧̃̃es asilares filantrópicas e particulares da grande Florianópolis [dissertação de mestrado]. Florianópolis: Universidade do Estado de Santa Catarina. 2004. 